\title{
The Impact of Prostate Cancer Treatment on Quality of Life: A Narrative Review with a Focus on Randomized Data
}

This article was published in the following Dove Press journal: Research and Reports in Urology

\author{
James M Taylor \\ Victor E Chen \\ Ryan C Miller \\ Benjamin A Greenberger (D) \\ Department of Radiation Oncology, \\ Sidney Kimmel Medical College \& Cancer \\ Center at Thomas Jefferson University, \\ Philadelphia, PA, USA
}

\begin{abstract}
Despite excellent oncologic outcomes, the management of localized prostate cancer remains complex and is dependent on multiple factors, including patient life expectancy, medical comorbidities, tumor characteristics, and genetic risk factors. Decades of iterative clinical trials have improved the optimization and utilization of surgical and radiation-based modalities, as well as their combinatorial use with anti-androgen and systemic therapies. While cure rates are high and converging on equivalent disease control should an upfront surgical or radiotherapeutic approach be optimized, the long-term side effects of surgical and radiation-based treatments can differ significantly in nature. Decisions regarding the selection of therapy are therefore best made in an informed and shared medical decisionmaking process between clinician and patient with respect to cancer control as well as adverse effects. We outline in this narrative review an understanding regarding implications of surgical and radiation treatment on quality of life after treatment, and how these data may be considered in the context of advising patients regarding the selection of therapy. This narrative review largely focuses on the quality of life data obtained from prospective randomized trials of men treated for prostate cancer. We believe this provides the best assessment of the quality of life and can be used to inform patients when making treatment decisions.
\end{abstract}

Keywords: prostate cancer, radical prostatectomy, radiation therapy, quality of life, toxicity

\section{Approaches for Clinically Localized Prostate Cancer}

Estimated 191,930 new cases of prostate cancer will be diagnosed in the United States in 2020, accounting for $21 \%$ of new cancer cases in men. ${ }^{1}$ Overall prognosis, especially in the setting of localized disease, is extremely good with five-year relative survival greater than 98\% for all races between 2009 and 2015. In general, men who are older and have very low-risk prostate cancer may not benefit from active intervention and instead may choose a strategy of watchful waiting ${ }^{2}$ (Table 1). For men who have longer life expectancy and low-risk disease, active surveillance, radical prostatectomy (RP), external beam radiation (XRT), and brachytherapy (BT) are all options supported by the National Comprehensive Cancer Network (NCCN) guidelines. ${ }^{3-7}$ With regard to the radiation treatment course, conventional fractionation, hypofractionation, and stereotactic body radiotherapy (SBRT) may all be considered options based upon individual risk assessment, as well as tumor and treatment characteristics. ${ }^{8-12}$ NCCN favorable intermediate-risk disease $(<50 \%$ of cores positive, no Gleason Grade Group 3 or higher histology,
Correspondence: Benjamin A

Greenberger

Email benjamin.a.greenberger@gmail.com

Research and Reports in Urology 2020:12 533-546 
Table I Treatment Selection for Localized Prostate Cancer, Stratified by Risk Group

\begin{tabular}{|l|l|}
\hline Disease Group & Treatment Modalities \\
\hline Low-Risk & $\begin{array}{l}\text { If expected patient survival } \geq 10 \text { years, active surveillance, external beam radiation (standard fractionation, } \\
\text { hypofractionation, or SBRT), brachytherapy (HDR or LDR), and radical prostatectomy are all options; if < I0 years, } \\
\text { observation is recommended }\end{array}$ \\
\hline $\begin{array}{l}\text { Favorable Intermediate- } \\
\text { Risk }\end{array}$ & $\begin{array}{l}\text { If expected patient survival } \geq 10 \text { years, active surveillance, external beam radiation, brachytherapy, and radical } \\
\text { prostatectomy are all options; if < } 10 \text { years, observation, external beam radiation, or brachytherapy is recommended }\end{array}$ \\
\hline Intermediate-Risk & $\begin{array}{l}\text { If expected patient survival } \geq 10 \text { years, radical prostatectomy, external beam radiation with 4-6 months of ADT, } \\
\text { external beam radiation and brachytherapy boost and 4-6 months of ADT are all options; if < 10 years, observation, } \\
\text { external beam radiation with 4-6 months ADT, and external beam radiotherapy and brachytherapy boost and 4-6 } \\
\text { months ADT are all options }\end{array}$ \\
\hline High-Risk & $\begin{array}{l}\text { If }>5 \text { years or symptomatic, external beam radiation with I8-36 months ADT, external beam radiation and } \\
\text { brachytherapy boost and I8-36 months of ADT, and radical prostatectomy are all options; if } \leq 5 \text { years and } \\
\text { asymptomatic, observation, external beam radiation and ADT are all options }\end{array}$ \\
\hline Adjuvant/Salvage & $\begin{array}{l}\text { If adverse features (positive margins, seminal vesicle involvement, and/or extraprostatic extension), consider adjuvant } \\
\text { external beam radiation; otherwise, close monitoring with external beam radiation and 6-24 months of ADT } \\
\text { recommended if clinical or biochemical evidence of failure }\end{array}$ \\
\hline
\end{tabular}

only one intermediate-risk factor) has a treatment algorithm that is similar to low-risk disease. ${ }^{13}$ Unfavorable intermediate-risk and high-risk diseases typically require the addition of androgen deprivation therapy (ADT), as well as potentially a brachytherapy boost, to external beam radiotherapy. ${ }^{6,14-20}$ Regarding length of ADT, unfavorable intermediate-risk disease and high-risk disease typically require 4-6 months and 18-36 months, respectively, with currently less level 1 data regarding justifying the optimal length of ADT in the setting of brachytherapy boost. ${ }^{6,21-25}$ The addition of systemic therapy, e.g. docetaxel, to radiation has been historically more controversial and individualized in the context of high-risk, localized disease, though recent data from the GETUG 12 trial as well as RTOG 0521 have suggested superior 8-year relapse-free survival and 4-year overall survival, respectively. ${ }^{26,27}$ Given the limited data, however, the addition of docetaxel to ADT plus XRT in high- or very high-risk prostate cancer is not categorically recommended as of NCCN Version 2.2020 until longer follow-up demonstrates comparable success of salvage treatments and rates of longterm adverse effects.

External beam radiation often incorporates pelvic nodal treatment when there is a threshold risk of lymph node involvement, e.g. $15 \%$ or higher ${ }^{28,29}$ (Figure 1). This risk is estimated based on clinical factors such as Gleason Score (GS) and Prostate Specific Antigen (PSA), often with the assistance of a nomogram. RTOG 0924 trial is underway to provide further insight into the definitive RT setting. Postoperatively, XRT and ADT still may be a requirement in the adjuvant setting depending on factors such as lymph node involvement, positive margins, extraprostatic extension, and seminal vesicle involvement. ${ }^{30-34}$ If adjuvant treatment is not pursued based on favorable pathology, salvage radiation to the disease bed or pelvis, as well as ADT, still is required if there is clinical or biochemical evidence of failure, ${ }^{35,36}$ with the extent of nodal treatment additionally under investigation in the salvage setting. ${ }^{37}$

The first generation of meta-analyses utilizing observational data compared surgical and radiation-based modalities across all risk groups of localized disease with regard to overall mortality (OM) and prostate cancer-specific mortality (PCM) hazard ratios, the most well known of which found OM and PCM benefits to upfront surgery across all groups. ${ }^{38}$ Additional efforts found that controlling for quality of ADT, adequate radiation dose, and overall compliance with modern NCCN standard-ofcare diminished these differences. ${ }^{39,40}$ There is even a suggestion of improved PCM for RT-based modalities compared with RP in the setting of Gleason 9-10 disease when BT is used with XRT, though there are limited observational data available to answer this question. ${ }^{41-}$ ${ }^{44}$ There is some evidence that this advantage for brachytherapy boost diminishes in the setting of RP plus adjuvant RT. ${ }^{43,44}$ Should these data prove verifiable 


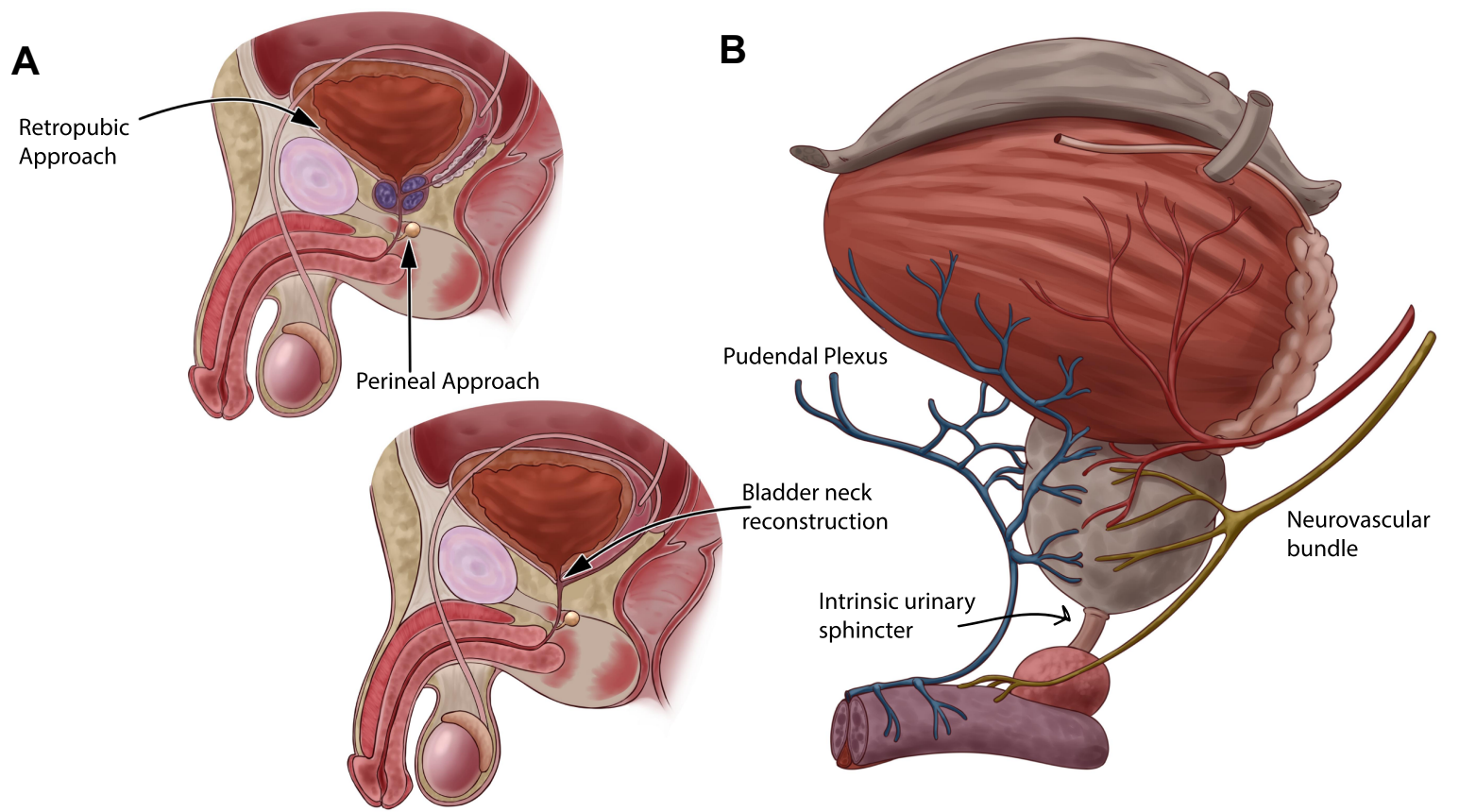

C
Intact Prostate + SV CTV
Pelvic lymph node XRT CTV (if indicated)

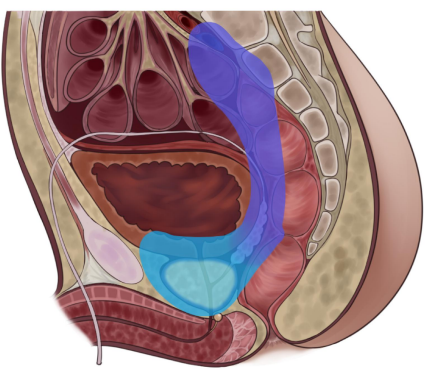

D
Prostate fossa CTV

Pelvic lymph node XRT CTV (if indicated)

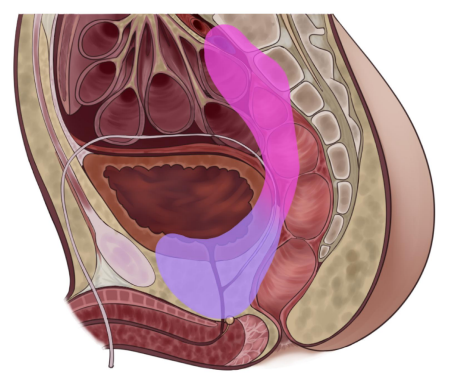

E

- radioactive seed (e.g., I-125, Pa-103, Cs-131)

Intact prostate CTV

Pelvic lymph node XRT CTV (if indicated)

Figure I Surgical and Radiotherapeutic Approaches in the Treatment of Prostate Cancer. (A) pre- and post-prostatectomy anatomy with retropubic and perineal surgical approaches. (B) microsurgical anatomy at risk for injury with retropubic and surgical approaches. (C) clinical target volumes (CTV) for radiotherapy for intact prostate treated definitively with external beam radiotherapy (XRT). When indicated pelvic lymph node fields are added, increasing the potential for gastrointestinal toxicity. (D) CTV for radiotherapy for prostate fossa treated adjuvantly or in salvage cases. The utility of lymph node coverage in this setting is the subject of clinical investigation. (E) CTV for low-dose-rate brachytherapy with or without XRT treatment. High-dose-rate techniques are available using temporary catheter-based placement of an Ir-192 source with established fractionation schedules and fewer post-treatment radiation precautions.

with prospective data, there may be a subset of patients with Gleason 9-10 or other high-risk diseases for which a discussion of treatment options may converge on XRT $+\mathrm{BT}$ with ADT versus RP with a very strong possibility of requiring adjuvant RT. Counseling patients regarding the burden of GU-related toxicity associated with BTboost is substantially different if the pre-procedural probability is high that adverse features will prompt adjuvant radiation to the prostatic fossa and possibility pelvic lymph nodes.
Given the high rates of success for oncologic outcomes as well as more robust algorithms to achieve similar oncologic outcomes with upfront RP or RT, there is a need to better understand the toxicities of various treatment modalities and how they affect patient quality of life. To help providers with this important conversation, we have provided a narrative review of the data involving treatment-related toxicity and potential interventions to ameliorate toxicity during and after treatment. 


\section{Methods}

We performed a search of the PubMed database to identify pertinent articles that included quality of life assessments relating to the treatment of prostate cancer and common treatment modalities including RP, XRT, BT, and ADT. The search focused on randomized clinical trials comparing treatment modalities for prostate cancer that included quality of life metrics. In addition to database searches, hand searches were also conducted based on expert opinion and author consensus. Studies including intraoperative fresh-frozen pathologic analysis of margins to improve nerve sparing during RP or injection of hydrogel spacers to improve rectal sparing during RT were not included, given their relative novelty and lack of randomized data comparing surgical and radiotherapeutic outcomes. Searches of published abstracts were also included in the formation of the manuscript when full articles were not available. Finally, the manuscript and reference list were evaluated and approved by all authors for inclusion in the current publication.

\section{Technical Approaches for Surgery and Radiation}

In general, there are two common approaches to RP, one which employs a retropubic approach, in which the prostate is removed through an incision in the wall of the abdomen, and the other a perineal approach, in which the incision is made in the area between the scrotum and anus $^{45}$ (Figure 1). Retropubic prostatectomy was considered the gold standard since the early 1980s due to the ability to access and remove pelvic lymph nodes, as well as spare cavernosal nerves. The dorsal vein complex is at risk, however, and blood loss requiring transfusions has been shown to be a risk of this approach. ${ }^{46}$ Since many patients at low risk for metastasis do not require extensive pelvic lymph node dissection, a perineal prostatectomy is an alternative approach and often causes less blood loss by avoiding the dorsal vein complex. ${ }^{47}$ Regarding robotic prostatectomy, studies have shown lower estimated blood loss, shorter hospital stays, a lower incidence of bladder neck contractures/anastomotic strictures, and lower intraoperative adverse event. ${ }^{48}$ However, there is a lack of randomized, long-term studies that compare functional and oncologic outcomes between open and robotic approaches. Modern robotic techniques (e.g., NeuroSAFE) continue to evolve, including intraoperative fresh-frozen section analysis of prostate margins to assess and optimize oncologic resection while maintaining functional outcomes, with randomized trials underway. ${ }^{49}$ Overall, most patients who opt for definitive management with prostatectomy can initially avoid androgen deprivation therapy and its associated effects on quality of life (QOL).

With regard to radiation, there are multiple techniques including XRT and BT. Dose escalation to 74-81 Gy has been shown to improve biochemical failure-free survival over lower doses; however, no overall survival benefit has been shown with these increasing doses over lower doses ( 70 Gy).$^{8,50-53}$ Hypofractionation, which typically uses 60-70 Gy over a shorter time interval, has demonstrated noninferiority to standard fractionation for low, intermediate, and high-risk disease; SBRT, a form of ultra-hypofractionated RT using image guidance and highly conformal techniques, has appeared safe at currently available follow-up and has allowed select patients to finish radiotherapy in 2 weeks. ${ }^{54}$ Treatment to the prostate in the definitive setting is delivered to the organ with an additional 5-10 $\mathrm{mm}$ margin to account for organ motion or setup uncertainties, with the extent of coverage of the seminal vesicles informed by risk group and several intensity-modulated radiotherapy protocols available to guide planning. ${ }^{55}$ Surrounding organs, such as the bladder, rectum, penile bulb, and bilateral femora, are delineated during planning as they receive radiation dose during treatment. ${ }^{56}$ In the adjuvant and salvage setting, the prostate fossa is typically defined as at least $2 \mathrm{~cm}$ above the pubic symphysis to the top of the penile bulb, including surgical clips and seminal vesicle remnants if involvement is detected, with an additional $8-15 \mathrm{~mm}$ margin added due to setup uncertainty and movement. ${ }^{57}$ The decision to treat lymph nodes significantly affects the anticipated exposure to the rectum and surrounding organs, as does the amount of margin necessary in the setting of chosen technique and image guidance. Rectal spacers are often used to mitigate short and late-term side effects of radiation therapy, ${ }^{58}$ though these techniques have not yet been reflected in randomized data comparing the quality of life following surgery versus radiation.

\section{Limited Conclusions from Retrospective Observational Data}

The reference standard in determining the impact any treatment modality has on an individual patient is the use of patient-reported outcomes (PROs), as they represent the 
patients' perspective on how their quality of life has changed in contrast to physician-graded toxicity. ${ }^{59}$ Reportable tools and assessments have been developed and increasingly deployed across observational and prospective research. ${ }^{60}$ Given the sample sizes and amount of followup required to measure differences in both frequent and infrequent morbidity endpoints, it has been tempting to use prostate cancer registries to answer questions regarding the differential incidence of various toxicities between approaches using upfront RP and RT. These cancer registries have tracked different levels of detail concerning treatment, with several important features such as ADT use, RT dose, and radiation technique inconsistently tracked and reported. ${ }^{61}$ Moreover, the use of these registries has more categorially been challenged by data suggesting that the systematic concordance between hazard ratios generated from observational studies and corresponding randomized controlled trials is little better than if left to chance. ${ }^{62}$

Despite these caveats, multiple efforts have attempted to leverage large sample size and registry data to answer quality of life questions following prostate cancer treatment. ${ }^{63-66}$ The Prostate Cancer Outcomes Study utilized six Surveillance, Epidemiology and End Results registries to recruit 3533 men, 1655 of whom had surgery or radiotherapy for localized disease within a year of diagnosis with follow-up surveys at 2 and 5 years. ${ }^{63}$ Men following surgery demonstrated more urinary incontinence and erectile dysfunction, while those receiving radiotherapy predominantly demonstrated GI symptoms including bowel urgency. These symptoms were noted at the early time points of 2 and 5 years, though all differences save for bowel symptoms were insignificant by year 15 . Most of these men notably received an older generation of radiation therapy, with inconsistent utilization of ADT, though more modern data have been utilized to replicate these findings. ${ }^{64,65}$

\section{Quality of Life Outcomes from Randomized Data and Prospective Cohort Studies}

Prospective randomized controlled trials (PRTs) are considered by many as the reference standard for clinical studies as they allow for equal distribution of both measured and unmeasured covariates across strata of control and exposure groups, create a baseline timepoint for cohort evaluation and lend to easy statistical analysis. It has become increasingly important to also assess functional outcomes when designing and conducting clinical trials in men with prostate cancer due to the improved understanding of converging oncologic outcomes with surgery and radiation. Accordingly, it would reason that PROs incorporated into prospective randomized studies of men treated for prostate cancer would serve as the highest level of evidence to directly compare treatment-related toxicity and QOL. Despite this, there remains a paucity of PROs obtained from RCT's to help guide physicians and patients on expected toxicities when selecting an appropriate treatment approach in the setting of multidisciplinary care.

The quality of life companion article of men enrolled in the Prostate Testing for Cancer and Treatment (ProtecT) trial may provide the best prospective QOL data in men with localized prostate cancer following treatment with one of the most commonly used methods: surgery, radiotherapy or active monitoring. ${ }^{67}$ Briefly, the ProtecT study was a multicenter, randomized clinical trial of men with screening-detected prostate cancer who were randomized in a 1:1:1 fashion to either radical prostatectomy, active monitoring or radiotherapy with 4 months of ADT. ${ }^{4}$ In all, 545 men were randomized to active monitoring, 553 men to radical prostatectomy and 545 men to radiotherapy + ADT. After 10 years of follow-up, the authors noted no difference in PCM, though the measured PCM remained low in all cohorts, with only 17 prostate cancer-specific deaths. ${ }^{4}$ It is important to note that most men enrolled in the ProtecT study had low-risk disease and further extrapolation of this study to cohorts of men with high-risk disease is cautioned, a risk category that will likely need to wait for the results of SPCG-15 for randomized data. ${ }^{68}$

In the ProtecT QOL study by Donovan et al, 1643 men enrolled on ProtecT completed validated questionnaires before diagnosis, at 6 and 12 months after randomization, and annually thereafter. ${ }^{67}$ The companion study was designed to provide a direct comparison of the QOL of men treated uniformly with either radiotherapy/ADT, radical prostatectomy, or active monitoring. The study included validated measures that assessed multiple domains including urinary, bowel, sexual function, and specific effects on quality of life, anxiety, depression and general health. The published report included 5 years of follow-up data on cancer-related quality of life and 6 years of follow-up data on additional endpoints. The authors analyzed the data according to the intention-to-treat principle based upon patient randomization into the treatment 
groups of the ProtecT trial. Overall, radical prostatectomy was associated with worse outcomes in erectile dysfunction and urinary incontinence compared with radiotherapy and 4 months of ADT. Conversely, radiotherapy and ADT were associated with significantly more bowel toxicity (Table 2). Importantly, when assessing the entire cohort, no significant differences were observed among any treatment group in general health-related, cancer-related quality of life, depression or anxiety. ${ }^{67}$ Despite equivalence in global health-related quality of life between treatment modalities, the study does demonstrate that toxicity profiles between radiotherapy and radical prostatectomy are different. These data are useful when discussing with patients. Overall, radiotherapy is more associated with worsened bowel symptoms while prostatectomy is associated with worsening of urinary incontinence and erectile dysfunction. To magnify this association, the authors report that compared to the active monitoring group the number needed to treat (NNT) to cause a single additional case of erectile dysfunction at 2 years is 4 with radical prostatectomy and 8 with radiotherapy, and the NNT to cause a single additional case of urinary incontinence at 2 years is 5 for RP and 143 for RT. However, as follow-up continued to 6 years the authors demonstrate that urinary and sexual function had stabilized in the radiotherapy group after improving for 2-3 years, while urinary and sexual function declined over this time period in the active monitoring group. As such, with this additional follow-up, the authors conclude that men treated with either active monitoring or radiotherapy with ADT had similar QOL with respect to sexual function and urinary incontinence, but these scores remained worse in men treated with prostatectomy.

It is important to note that the ProtecT study used modern approaches to surgery and thus may not be biased by older surgical procedures. The authors note that the majority of the 545 men randomized to radical prostatectomy underwent an open retropubic, nerve-sparing procedure. This nerve-sparing approach would be expected to reduce the rates of toxicities such as erectile dysfunction. In the radiation arm, patients were treated with modern dose-escalated prescriptions to $7400 \mathrm{cGy}$ in 37 fractions using three-dimensional conformal radiotherapy. While the dose in this cohort reflects modern prescribing, newer techniques such as intensity-modulated radiotherapy (IMRT) or volumetric modulated arc therapy (VMAT) are now more routinely used as they are associated with fewer toxicities compared with $3 \mathrm{D}$ conformal radiation. ${ }^{69}$
Despite this, the QOL data from the ProtecT study provide high-quality evidence regarding the impact of modern treatments for men with clinically localized prostate cancer and can be used to guide physicians and patients when considering treatment recommendations.

Another PRT comparing surgery vs radiotherapy with PROs was conducted by Lennernas et al and included men with locally advanced high-risk prostate cancer. ${ }^{70}$ This study conducted in Sweden between 1996 and 2001 included 89 men and randomized them 1:1 to either radical prostatectomy or radiotherapy consisting of 50 Gy external beam radiotherapy with high-dose-rate brachytherapy delivered in 2 separate fractions of 10 Gy each. All men in the trial were also treated with 6 months of neoadjuvant total androgen blockade. The primary outcome was the difference in health-related quality of life (HRQoL) using PROs. The study assessed self-reported HRQoL and symptoms including urinary, bowel, and sexual function prior to randomization and at 12 and 24 months following randomization. ${ }^{70}$ The authors report that no discernible differences in HRQoL or complications were demonstrated between the two treatment groups. One unique aspect of the study is that all men were treated with total androgen blockade. This allows for a better comparison between the two treatment modalities of radiotherapy and surgery without ADT confounding the radiotherapy arm. It also compares what has been described as maximal radiotherapy with combination XRT and BT. Combination XRT and BT have been shown to be associated with significantly more toxicity compared with XRT alone in prospective studies. ${ }^{20}$ Given that all men were treated with ADT and the radiotherapy arm received maximal radiotherapy, this study provides data that even in high-risk men treated aggressively, HRQoL as measured using PROs was similar between treatment arms. This study is limited by the small sample size and the nonconventional XRT dose given in the radiation arm but still provides valuable information using PROs from an RCT.

Another (pseudo)randomized study that published PROs is the American College of Surgeons Oncology Group Phase III Surgical Prostatectomy versus Interstitial Radiation Intervention Trial (ACOSOG SPIRIT) by Crook et al. ${ }^{71}$ This study compared RP with BT given definitively as monotherapy. The trial was initially designed as a randomized study but after slow accrual, the protocol was amended to allow patients to choose or be randomized to either RP or BT. Overall, the trial was terminated early due to poor accrual but the authors report on HRQoL at 5 


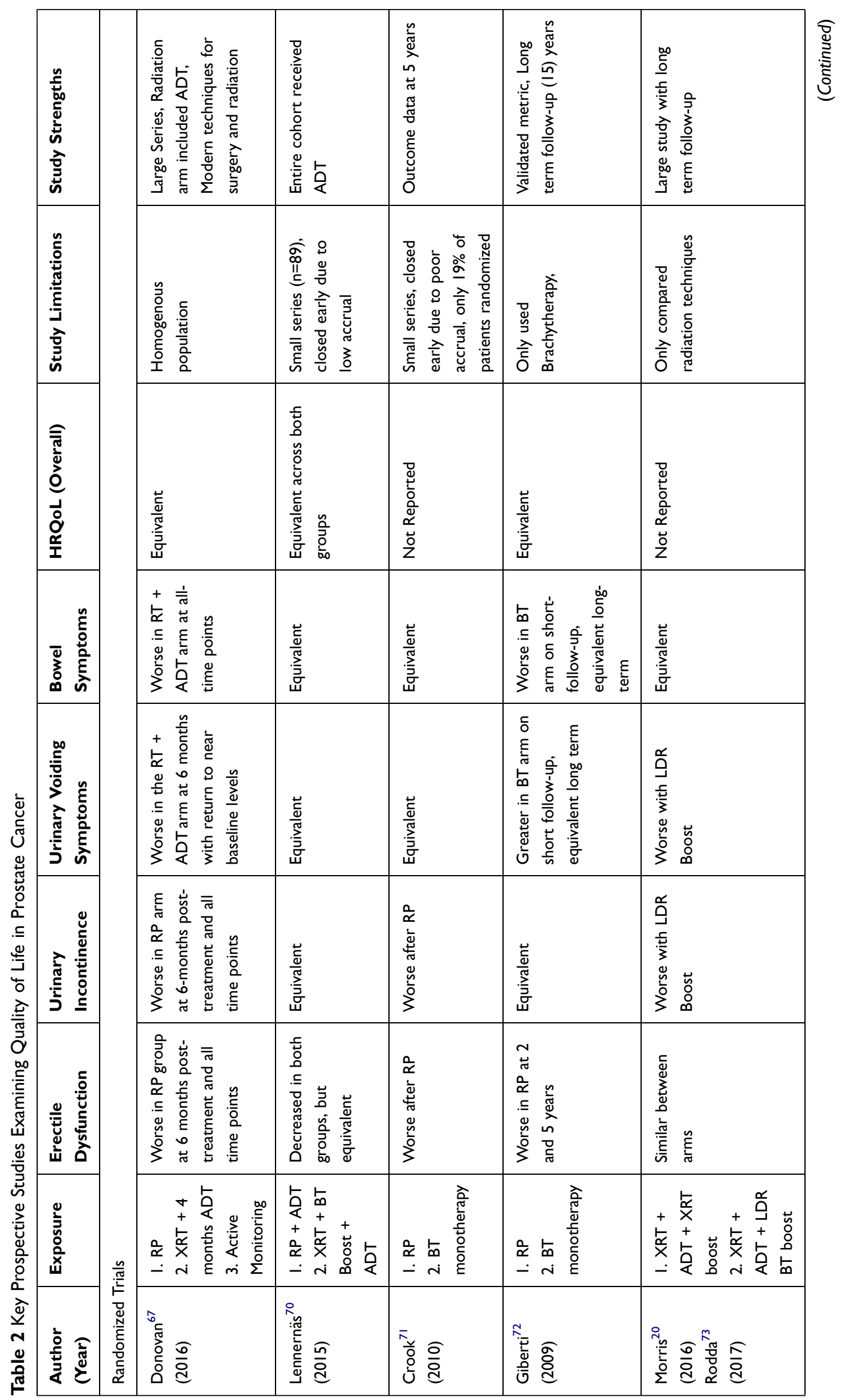




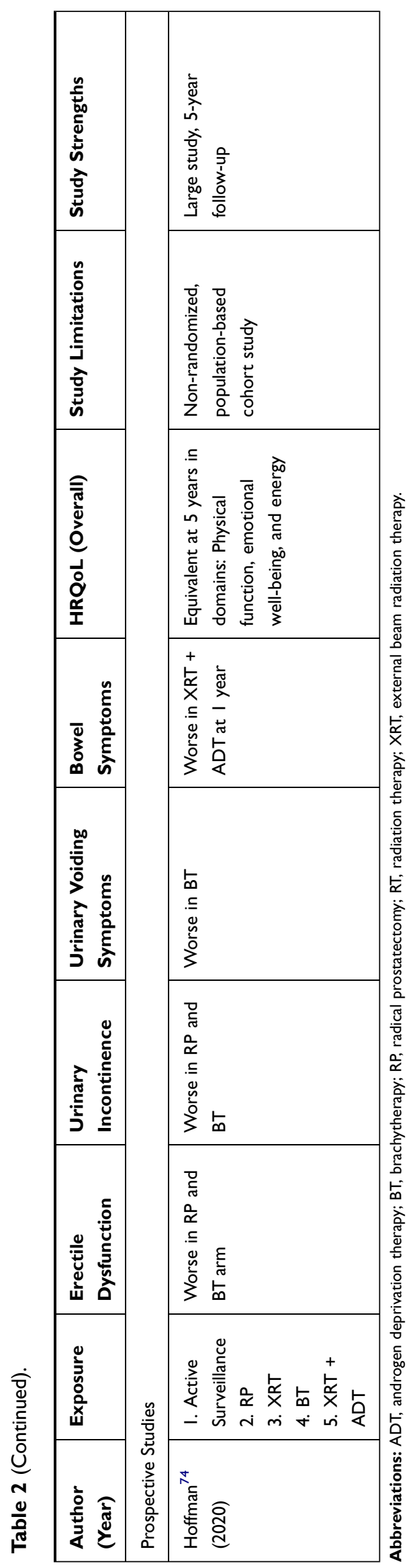

years in patients enrolled on the trial. Overall, 168 men were eligible for the toxicity analysis. In the entire cohort, $60.7 \%$ of men had BT (9.5\% randomly assigned) and $39.3 \%$ of men had an RP (9.5\% randomly assigned). Five years after treatment all men were considered for HRQoL evaluation using the cancer-specific 50-item Expanded Prostate Cancer Index Composite, the Short Form 12 Physical Component Score, and the Short Form 12 Mental Component Score. The overall response rate was $88.4 \%$. With a median follow-up of 5.2 years, the authors report no differences between RP and BT in bowel or hormonal domains. However, men treated with BT scored better in urinary $(91.8 \mathrm{v} 88.1 ; \mathrm{p}=0.02)$ and sexual $(52.5 \mathrm{v} 39.2 ; \mathrm{p}=0.001)$ domains as well as in patient satisfaction (93.6 v 76.9; $\mathrm{P}<0.001) .{ }^{71}$ This study is limited in its small sample size and largely non-random allocation (only 19\% of patients were randomly assigned) but provides evidence that brachytherapy as monotherapy had fewer adverse effects on HRQoL compared with prostatectomy.

In a similar study, Giberti et al randomized 200 patients to either radical retropubic prostatectomy or brachytherapy as monotherapy and reported on oncological and functional outcomes. ${ }^{72}$ Men randomized to surgery underwent bilateral nerve-sparing retropubic RP and standard lymph node dissection and men randomized to $\mathrm{BT}$ received lowdose rate brachytherapy using I-125 seeds with a prescribed dose of D90 > 140 Gy. All men received questionnaires including the International Prostate Symptom Score (IPSS), International Index of Erectile Function (IIEF), and the EORTC-QLQ-C30/PR25. Patients enrolled in the study completed questionnaires before treatment as well as every 3 months for the first year, every 6 months for the second year, and then annually. In this study, the authors report that RP was associated with worse erectile function while BT was associated with worse urinary voiding symptoms and bowel symptoms. Unlike the previously discussed studies, the authors found no difference in rates of urinary incontinence between study arms. Finally, as demonstrated in several other PRTs with PROs the authors report equivalent HRQoL between the treatment arms. ${ }^{72}$

Although not a trial of radiation compared with prostatectomy, the Androgen Suppression Combined with Elective Nodal and Dose Escalated Radiation Therapy (ASCENDE-RT) trial was a PRT of men with localized prostate cancer randomized to two different radiation techniques. $^{20}$ The study enrolled 398 men all of whom 
received 12 months of neoadjuvant ADT followed by XRT to the prostate and elective nodes to a total dose of $46 \mathrm{~Gy}$. Men were randomized into one of the two radiation boost arms: a standard dose-escalated XRT boost of an additional 32 Gy to a total of 78 Gy, or an LDR brachytherapy implant. The authors conclude that with 9 years of followup patients treated with an LDR brachytherapy implant had improved biochemical progression-free survival. However, compared with men who only received an XRT boost, those who underwent LDR brachytherapy had worse GU and GI toxicity as graded using the modified Late Effects of Normal Tissue - Somatic, Objective, Management, Analytic (LENT-SOMA) scale. ${ }^{73}$ Although not an RCT between surgery and radiotherapy this study provides evidence that XRT with ADT combined with an LDR brachytherapy procedure has greater toxicity compared with the more commonly used approach of ADT with dose-escalated external beam radiotherapy.

A recently published prospective cohort study by Hoffman et al detailed the QOL impact of 1386 men with favorable-risk localized prostate cancer treated with either active surveillance $(n=363)$, nerve-sparing prostatectomy $(n=675)$, XRT alone $(n=261)$ or low-dose rate brachytherapy $(n=87)$ and in men with unfavorable-risk localized prostate cancer treated with RP $(n=402)$ or XRT + ADT $(n=217) .{ }^{74}$ Patients reported their function using the 26-item Expanded Prostate Index Composite 5 years after completing treatment. Among men with favorable-risk disease, nerve-sparing prostatectomy was associated with worse urinary continence at 5 years and sexual function at 3 years compared with active surveillance. XRT was not associated with worse bowel, urinary or sexual side effects compared with active surveillance at any point during 5 years after treatment. In the unfavorable risk subset, XRT + ADT was associated with worse bowel function at 1 year, but better sexual function and incontinence compared with RP at each time point through 5 years. Overall, the results of the Hoffman analysis provide evidence that XRT has a more favorable toxicity profile compared with RP even when ADT is added in the unfavorable subgroup. Fortunately, none of the treatment groups reported meaningful declines in the HRQOL domains of physical function, emotional well-being, and energy/fatigue scores.

As discussed, ADT is often added to XRT or BT in unfavorable intermediate-risk and high-risk patient populations. In addition to the toxicities caused by radiotherapy, ADT introduces additional side effects. Several studies have shown that ADT independent of the effects of radiotherapy contributes to worse sexual function. ${ }^{75,76}$ In a study by Gay et al the addition of neoadjuvant ADT to XRT or BT was associated with worse QOL. Specifically, the authors demonstrate that men on ADT had a decreased ability to reach orgasm, had decreased quality of erections and decreased sexual function. ${ }^{77}$ ADT also has metabolic effects and can lead to increased weight gain and loss of muscle mass. Unlike other toxicities associated with prostate cancer treatment, the side effects attributable to ADT are often only transient and resolve after discontinuation and normalization of testosterone levels.

Finally, in men who undergo RP but require postoperative radiotherapy either in the adjuvant or salvage setting the addition of radiotherapy after prostatectomy has been shown to worsen QOL. Jenkins et al studied 106 men with $\mathrm{PCa}$ receiving post-prostatectomy radiotherapy and assessed the QOL impact of radiotherapy using the Expanded Prostate Cancer Index Composite (EPIC). The authors demonstrate that post-prostatectomy radiotherapy worsened urinary QOL with the most significant decline noted in urinary incontinence. ${ }^{78}$ Other studies of postprostatectomy radiotherapy show only temporary worsening of symptoms in urinary, sexual and bowel domains. ${ }^{79}$ Overall, studies seem to indicate a worse urinary function in men who require post-prostatectomy radiotherapy. ${ }^{80}$ Given this information, it is important to consider these implications when counseling men with high risk, high volume disease (Gleason score 9-10) who would most likely require adjuvant/salvage radiotherapy following RP. In this setting, men may wish to consider definitive $\mathrm{XRT}+\mathrm{ADT}$ and forgo RP given the high chances of requiring additional radiotherapy and the negative QOL impacts demonstrated in men receiving both modalities of treatment.

\section{Managing Toxicities Associated with Prostate Cancer Treatments}

Given how successful treatment generally is for localized prostate cancer, managing the adverse effects associated with the various available treatment strategies is of paramount importance in order to maximize patient quality-oflife. For example, it is well known that one of the most bothersome toxicities associated with a surgical approach to prostate cancer treatment is urinary incontinence, with the risk of incontinence being highly dependent on both age and surgical technique. ${ }^{81}$ Multiple strategies to aid 
men to recover urinary control have been investigated, including both procedural and non-procedural techniques. For example, placement of artificial urinary sphincters has been shown to be a viable option, with the caveat that late complications may occur which may require further procedural intervention to remove the device. ${ }^{82}$ In addition, transobturator sling suspension via placement of transobturator tape has also been studied, resulting in a significant rate of both complete cure of incontinence and decreased pad usage. ${ }^{83}$ Finally, behavioral training with biofeedback seems to be effective specifically for stress and urge incontinence, but less so with patients who experience continual leakage of urine following a prostatectomy. ${ }^{84}$

While incontinence following radiation treatment for prostate cancer is a rare side effect, radiation may instead cause hemorrhagic cystitis and radiation proctitis, both of which also negatively impact patient quality of life. Radiation cystitis can manifest itself both in the acute post-treatment time period and multiple months to years following completion of radiation treatment, and can vary significantly in severity from a mild bleed up to significant hemorrhage; as such, management strategies differ but include cystoscopy with electrocoagulation of bleeding areas, injection of botulinum toxin into the bladder wall, and instillation of compounds such as formalin or prostaglandins into the bladder itself. ${ }^{85}$ For radiation proctitis, direct instillation of formalin during a sigmoidoscopy has shown to be effective and often results in immediate resolution of symptoms. ${ }^{86,87}$ Alternatively, argon plasma coagulation, also performed during a flexible sigmoidoscopy, has also been demonstrated to be effective in both reducing blood transfusion requirements related to radiation proctitis and improving other aspects of bowel function such as resolving rectal urgency and stool frequency. ${ }^{88}$ Radiofrequency ablation is an option for those whose symptoms do not resolve with first or second-line interventions as well. ${ }^{89}$ For those who prefer a less invasive therapy, short-chain fatty acid enemas were superior to placebo in a prospective, randomized doubleblind trial examining the rapidity of healing of radiation proctitis. ${ }^{90}$ Finally, hyperbaric oxygen has been shown to be an effective treatment strategy for both patient complaints of hematuria related to radiation cystitis and rectal bleeding or irritation from radiation proctitis following radiation treatment for prostate cancer, but the use of this technique is generally reserved after other lines of therapy have been ineffective. ${ }^{91,92}$
For many men undergoing treatment for prostate cancer, maintenance of sexual function is an extremely important consideration and component of their quality of life; unfortunately, sexual dysfunction is a common adverse effect of both RT and RP, with similar rates for both therapies (particularly now that surgical techniques have improved such that nerve-sparing approaches are possible for localized disease). Complicating studies on this subject is the fact that with the exception of a recently validated standardized questionnaire, the International Index of Erectile Function (IIEF), defining terms such as "potency," "satisfactory sexual function," "sexual interest," and "libido," among others, is dependent on individual interpretation. ${ }^{93}$ The first-line treatment for erectile dysfunction is generally an oral phosphodiesterase type 5 (PDE5) inhibitor, although specific dosages and frequencies of administration vary. ${ }^{94}$ In fact, prophylactic sildenafil citrate initiated before radiation therapy for prostate cancer and given continuously with the intent of preserving erectile function until 6 months following completion of radiation therapy was demonstrated to improve long-term sexual outcomes. ${ }^{95}$ Should patients not respond to oral PDE5 agents, patients are generally offered penile injections, penile implants, or vacuum erection devices. ${ }^{96-98}$ Less invasive options such as exercise therapy (with moderate to high intensity aerobic and resistance training exercises) have also been shown to be effective, even though they do not directly act on sexual organs, as sexual function is intimately interwoven with mental health and well-being. ${ }^{99}$ Finally, clear, constructive communication between patients and their partners has been consistently shown to be one of the most important factors in helping patients cope with sexual problems following prostate cancer treatment. ${ }^{100}$

Perhaps most importantly, comprehensive patient counseling of the risks and benefits of each potential treatment option is appropriate in men with localized prostate cancer. Many tools are available to aid this process; for example, nomograms have been developed to predict both acute and long-term rectal toxicity associated with radiation therapy ${ }^{101-103}$ as well as urinary adverse events. ${ }^{104}$ Involvement of a multidisciplinary team of healthcare providers is essential such that the patient is able to appreciate all available options for treatment, and ultimately, although a consensus recommendation can be made based on patient characteristics and/or disease involvement, patient participation with an informed final decision is the most critical component to the management of patient expectations regarding toxicity. ${ }^{105-107}$ 


\section{Conclusion}

As discussed, there is a paucity of prospective randomized data with PRO metrics for men treated with localized prostate cancer. Based on the current review it appears that prostatectomy is associated with higher rates of urinary incontinence and erectile dysfunction compared to radiotherapy. Unlike surgery, radiotherapy appears to be associated with worsening bowel function. However, despite these differences, prospective evaluation seems to indicate that global HRQoL does not differ in men by treatment modality. Thus, an individualized discussion that compares and contrasts different toxicity profiles of each treatment modality may be the best approach clinically to encourage patient engagement in the shared medical decision-making process. There are myriad tools and interventions, both procedural and non-procedural, available to address the sequelae of an upfront RP or RT-based approach. In the future, we believe that all men with prostate cancer should have an informed discussion in the setting of a multi-disciplinary setting to hear of all of the information available before deciding on a treatment approach.

\section{Acknowledgments}

The authors would like to thank Herbert Colunga for artistic contributions related to Figure 1.

\section{Disclosure}

The authors report no conflicts of interest for this work.

\section{References}

1. Siegel RL, Miller KD, Jemal A. Cancer statistics, 2020. CA Cancer J Clin. 2020;70(1):7-30. doi:10.3322/caac. 21590

2. Albertsen PC. Observational studies and the natural history of screendetected prostate cancer. Curr Opin Urol. 2015;25(3):232-237. doi:10.1097/MOU.0000000000000157

3. Bill-Axelson A, Holmberg L, Garmo H, et al. Radical prostatectomy or watchful waiting in early prostate cancer. $N$ Engl J Med. 2014;370 (10):932-942. doi:10.1056/NEJMoa1311593

4. Hamdy FC, Donovan JL, Lane JA, et al. 10-year outcomes after monitoring, surgery, or radiotherapy for localized prostate cancer. $N$ Engl J Med. 2016;375(15):1415-1424. doi:10.1056/NEJMoa1606220

5. Wilt TJ, Jones KM, Barry MJ, et al. Follow-up of prostatectomy versus observation for early prostate cancer. $N$ Engl J Med. 2017;377 (2):132-142. doi:10.1056/NEJMoa1615869

6. Mohler JL, Antonarakis ES, Armstrong AJ, et al. Prostate cancer, version 2.2019, NCCN clinical practice guidelines in oncology. J Natl Compr Canc Netw. 2019;17(5):479-505. doi:10.6004/jncen.2019.0023

7. Wallis CJD, Glaser A, Hu JC, et al. Survival and complications following surgery and radiation for localized prostate cancer: an international collaborative review. Eur Urol. 2018;73(1):11-20. doi:10.1016/j.eururo.2017.05.055
8. Dearnaley D, Syndikus I, Mossop H, et al. Conventional versus hypofractionated high-dose intensity-modulated radiotherapy for prostate cancer: 5-year outcomes of the randomised, non-inferiority, Phase 3 CHHiP trial. Lancet Oncol. 2016;17(8):1047-1060. doi:10.1016/S1470-2045(16)30102-4

9. Catton $\mathrm{CN}$, Lukka H, Gu C-S, et al. Randomized trial of a hypofractionated radiation regimen for the treatment of localized prostate cancer. J Clin Oncol. 2017;35(17):1884-1890. doi:10.1200/JCO.2016.71.7397

10. Lee WR, Dignam JJ, Amin MB, et al. Randomized phase III noninferiority study comparing two radiotherapy fractionation schedules in patients with low-risk prostate cancer. $J$ Clin Oncol. 2016;34(20):2325-2332. doi:10.1200/JCO.2016.67.0448

11. Widmark A, Gunnlaugsson A, Beckman L, et al. Ultra-hypofractionated versus conventionally fractionated radiotherapy for prostate cancer: 5-year outcomes of the HYPO-RT-PC randomised, non-inferiority, phase 3 trial. Lancet London Engl. 2019;394 (10196):385-395. doi:10.1016/S0140-6736(19)31131-6

12. King CR, Freeman D, Kaplan I, et al. Stereotactic body radiotherapy for localized prostate cancer: pooled analysis from a multi-institutional consortium of prospective Phase II trials. Radiother Oncol. 2013;109(2):217-221. doi:10.1016/j.radonc.2013.08.030

13. Zumsteg ZS, Spratt DE, Pei I, et al. A new risk classification system for therapeutic decision making with intermediate-risk prostate cancer patients undergoing dose-escalated externalbeam radiation therapy. Eur Urol. 2013;64(6):895-902. doi:10. 1016/j.eururo.2013.03.033

14. D'Amico AV, Manola J, Loffredo M, Renshaw AA, DellaCroce A, Kantoff PW. 6-month androgen suppression plus radiation therapy vs radiation therapy alone for patients with clinically localized prostate cancer: a randomized controlled trial. JAMA. 2004;292(7):821-827. doi:10.1001/jama.292.7.821

15. Pilepich MV, Winter K, Lawton CA, et al. Androgen suppression adjuvant to definitive radiotherapy in prostate carcinoma: longterm results of phase III RTOG 85-31. Int J Radiat Oncol Biol Phys. 2005;61(5):1285-1290. doi:10.1016/j.ijrobp.2004.08.047

16. Roach M, Bae K, Speight J, et al. Short-term neoadjuvant androgen deprivation therapy and external-beam radiotherapy for locally advanced prostate cancer: long-term results of RTOG 8610. J Clin Oncol. 2008;26(4):585-591. doi:10.1200/JCO.20 07.13.9881

17. Bolla M, Van Tienhoven G, Warde P, et al. External irradiation with or without long-term androgen suppression for prostate cancer with high metastatic risk: 10-year results of an EORTC randomised study. Lancet Oncol. 2010;11(11):1066-1073. doi:10.1016/S1470-2045(10)70223-0

18. Bolla M, Maingon P, Carrie C, et al. Short androgen suppression and radiation dose escalation for intermediate- and high-risk localized prostate cancer: results of EORTC trial 22991. J Clin Oncol. 2016;34(15):1748-1756. doi:10.1200/JCO.2015.64.8055

19. Nabid A, Carrier N, Vigneault E, et al. A phase III trial of shortterm androgen deprivation therapy in intermediate-risk prostate cancer treated with radiotherapy. J Clin Oncol. 2015;33 (15_suppl):5019. doi:10.1200/jco.2015.33.15_suppl.5019

20. Morris WJ, Tyldesley S, Rodda S, et al. Androgen suppression combined with elective nodal and dose escalated radiation therapy (the ASCENDE-RT trial): an analysis of survival endpoints for a randomized trial comparing a low-dose-rate brachytherapy boost to a dose-escalated external beam boost for high- and intermediate-risk prostate cancer. Int J Radiat Oncol. 2017;98(2):275-285. doi:10.1016/j.ijrobp.2016.11.026

21. Horwitz EM, Bae K, Hanks GE, et al. Ten-year follow-up of radiation therapy oncology group protocol 92-02: a phase iii trial of the duration of elective androgen deprivation in locally advanced prostate cancer. J Clin Oncol. 2008;26(15):2497-2504. doi:10.1200/JCO.2007.14.9021 
22. Bolla M, de Reijke TM, Van Tienhoven G, et al. Duration of androgen suppression in the treatment of prostate cancer. $N$ Engl J Med. 2009;12:2516-2527.

23. Pisansky TM, Hunt D, Gomella LG, et al. Duration of androgen suppression before radiotherapy for localized prostate cancer: radiation therapy oncology group randomized clinical trial 9910. $J$ Clin Oncol. 2015;33(4):332-339. doi:10.1200/JCO.2014.58. 0662

24. Denham JW, Steigler A, Lamb DS, et al. Short-term neoadjuvant androgen deprivation and radiotherapy for locally advanced prostate cancer: 10-year data from the TROG 96.01 randomised trial. Lancet Oncol. 2011;12(5):451-459. doi:10.1016/S1470-2045(11) 70063-8

25. Zapatero A, Guerrero A, Maldonado X, et al. High-dose radiotherapy with short-term or long-term androgen deprivation in localised prostate cancer (DART01/05 GICOR): a randomised, controlled, phase 3 trial. Lancet Oncol. 2015;16(3):320-327. doi:10.1016/S1470-2045(15)70045-8

26. Fizazi K, Faivre L, Lesaunier F, et al. Androgen deprivation therapy plus docetaxel and estramustine versus androgen deprivation therapy alone for high-risk localised prostate cancer (GETUG 12): a phase 3 randomised controlled trial. Lancet Oncol. 2015;16 (7):787-794. doi:10.1016/S1470-2045(15)00011-X

27. Rosenthal SA, Hu C, Sartor O, et al. Effect of chemotherapy with docetaxel with androgen suppression and radiotherapy for localized high-risk prostate cancer: the randomized phase III NRG oncology RTOG 0521 trial. J Clin Oncol. 2019;37(14):11591168. doi:10.1200/JCO.18.02158

28. Roach M, Moughan J, Lawton CAF, et al. Sequence of hormonal therapy and radiotherapy field size in unfavourable, localised prostate cancer (NRG/RTOG 9413): long-term results of a randomised, phase 3 trial. Lancet Oncol. 2018;19(11):1504-1515. doi:10.1016/S1470-2045(18)30528-X

29. Spratt DE, Vargas HA, Zumsteg ZS, et al. Patterns of lymph node failure after dose-escalated radiotherapy: implications for extended pelvic lymph node coverage. Eur Urol. 2017;71 (1):37-43. doi:10.1016/j.eururo.2016.07.043

30. Thompson IM, Tangen CM, Paradelo J, et al. Adjuvant radiotherapy for pathological T3N0M0 prostate cancer significantly reduces risk of metastases and improves survival: long-term followup of a randomized clinical trial. J Urol. 2009;181(3):956962. doi:10.1016/j.juro.2008.11.032

31. Wiegel T, Bartkowiak D, Bottke D, et al. Adjuvant radiotherapy versus wait-and-see after radical prostatectomy: 10-year followup of the ARO 96-02/AUO AP 09/95 trial. Eur Urol. 2014;66 (2):243-250. doi:10.1016/j.eururo.2014.03.011

32. Bolla M, van Poppel H, Tombal B, et al. Postoperative radiotherapy after radical prostatectomy for high-risk prostate cancer: long-term results of a randomised controlled trial (EORTC trial 22911). Lancet London Engl. 2012;380(9858):2018-2027. doi:10.1016/S0140-6736 (12)61253-7

33. Lawton CA, Winter K, Grignon D, Pilepich MV. Androgen suppression plus radiation versus radiation alone for patients with stage D1/pathologic node-positive adenocarcinoma of the prostate: updated results based on national prospective randomized trial Radiation Therapy Oncology Group 85-31. J Clin Oncol. 2005;23(4):800-807. doi:10.1200/JCO.2005.08.141

34. Tendulkar RD, Agrawal S, Gao T, et al. Contemporary update of a multi-institutional predictive nomogram for salvage radiotherapy after radical prostatectomy. J Clin Oncol. 2016;34(30):36483654. doi:10.1200/JCO.2016.67.9647

35. Shipley WU, Seiferheld W, Lukka HR, et al. Radiation with or without antiandrogen therapy in recurrent prostate cancer. $N$ Engl J Med. 2017;376(5):417-428. doi:10.1056/NEJMoa1607 529
36. Carrie C, Hasbini A, de Laroche G, et al. Salvage radiotherapy with or without short-term hormone therapy for rising prostate-specific antigen concentration after radical prostatectomy (GETUG-AFU 16): a randomised, multicentre, open-label phase 3 trial. Lancet Oncol. 2016;17(6):747-756. doi:10.1016/S1470-2045(16)00111-X

37. Pollack A, Karrison TG, Balogh AG, et al. Short term androgen deprivation therapy without or with pelvic lymph node treatment added to prostate bed only salvage radiotherapy: the NRG oncology/RTOG 0534 SPPORT trial. Int J Radiat Oncol. 2018;102 (5):1605. doi:10.1016/j.ijrobp.2018.08.052

38. Wallis CJD, Saskin R, Choo R, et al. Surgery versus radiotherapy for clinically-localized prostate cancer: a systematic review and meta-analysis. Eur Urol. 2016;70(1):21-30. doi:10.1016/j.eururo. 2015.11.010

39. Roach M, Ceron Lizarraga TL, Lazar AA. Radical prostatectomy versus radiation and androgen deprivation therapy for clinically localized prostate cancer: how good is the evidence? Int J Radiat Oncol. 2015;93(5):1064-1070. doi:10.1016/j.ijrobp.2015.08.005

40. Greenberger BA, Zaorsky NG, Den RB. Comparison of radical prostatectomy versus radiation and androgen deprivation therapy strategies as primary treatment for high-risk localized prostate cancer: a systematic review and meta-analysis. Eur Urol Focus. 2019. doi:10.1016/j.euf.2019.11.007

41. Greenberger BA, Chen VE, Den RB. Combined modality therapies for high-risk prostate cancer: narrative review of current understanding and new directions. Front Oncol. 2019;9. doi:10. 3389/fonc. 2019.01273

42. Kishan AU, Cook RR, Ciezki JP, et al. Radical prostatectomy, external beam radiotherapy, or external beam radiotherapy with brachytherapy boost and disease progression and mortality in patients with Gleason score 9-10 prostate cancer. JAMA. 2018;319(9):896-905. doi:10.1001/jama.2018.0587

43. Tilki D, Chen M-H, Wu J, et al. Surgery vs radiotherapy in the management of biopsy Gleason score 9-10 prostate cancer and the risk of mortality. JAMA Oncol. 2018. doi:10.1001/jamaoncol. 2018.4836

44. Muralidhar V, Mahal BA, Butler S, et al. Combined external beam radiation therapy and brachytherapy versus radical prostatectomy with adjuvant radiation therapy for Gleason 9-10 prostate cancer. J Urol. 2019;202(5):973-978. doi:10.1097/JU.000000 0000000352

45. Lepor H. A review of surgical techniques for radical prostatectomy. Rev Urol. 2005;7(2):S11-S17.

46. Koch MO, Smith JA. Blood loss during radical retropubic prostatectomy: is preoperative autologous blood donation indicated? $J$ Urol. 1996;156(3):1077-1080. doi:10.1016/S0022-5347(01)657 06-9

47. Lance RS, Freidrichs PA, Kane C, et al. A comparison of radical retropubic with perineal prostatectomy for localized prostate cancer within the Uniformed Services Urology Research Group: comparison of RRP with RPP for prostate cancer. BJU Int. 2001;87(1):61-65. doi:10.1046/j.1464-410x.2001.00023.x

48. Chandrasekar T, Tilki D. Robotic-assisted vs. open radical prostatectomy: an update to the never-ending debate. Transl Androl Urol. 2018;7(S1):S120-S123. doi:10.21037/tau.2017.12.20

49. Dinneen E, Haider A, Allen C, et al. NeuroSAFE robot-assisted laparoscopic prostatectomy versus standard robot-assisted laparoscopic prostatectomy for men with localised prostate cancer (NeuroSAFE PROOF): protocol for a randomised controlled feasibility study. BMJ Open. 2019;9(6):e028132. doi:10.1136/bmjopen-2018-028132

50. Kuban DA, Tucker SL, Dong L, et al. Long-term results of the M. D. anderson randomized dose-escalation trial for prostate cancer. Int J Radiat Oncol. 2008;70(1):67-74. doi:10.1016/j.ijrobp.2007. 06.054 
51. Zietman AL, Bae K, Slater JD, et al. Randomized trial comparing conventional-dose with high-dose conformal radiation therapy in early-stage adenocarcinoma of the prostate: long-term results from proton radiation oncology group/american college of radiology 95-09. J Clin Oncol. 2010;28(7):1106-1111. doi:10.1200/ JCO.2009.25.8475

52. Al-Mamgani A, van Putten WLJ, Heemsbergen WD, et al Update of dutch multicenter dose-escalation trial of radiotherapy for localized prostate cancer. Int J Radiat Oncol. 2008;72(4):980988. doi:10.1016/j.ijrobp.2008.02.073

53. Michalski JM, Moughan J, Purdy J, et al. Effect of standard vs dose-escalated radiation therapy for patients with intermediaterisk prostate cancer: the NRG oncology RTOG 0126 randomized clinical trial. JAMA Oncol. 2018;4(6):e180039. doi:10.1001/ jamaoncol.2018.0039

54. Roy S, Morgan SC. Hypofractionated radiotherapy for localized prostate cancer: when and for whom? Curr Urol Rep. 2019;20(9). doi:10.1007/s11934-019-0918-0

55. Fischer-Valuck BW, Rao YJ, Michalski JM. Intensity-modulated radiotherapy for prostate cancer. Transl Androl Urol. 2018;7 (3):297-307. doi:10.21037/tau.2017.12.16

56. Gay HA, Barthold HJ, O’Meara E, et al. Pelvic normal tissue contouring guidelines for radiation therapy: a Radiation Therapy Oncology Group consensus panel atlas. Int J Radiat Oncol. 2012;83(3):e353-e362. doi:10.1016/j.ijrobp.2012.01.023

57. Michalski JM, Lawton C, El Naqa I, et al. Development of RTOG consensus guidelines for the definition of the clinical target volume for postoperative conformal radiation therapy for prostate cancer. Int J Radiat Oncol. 2010;76(2):361-368. doi:10.1016/j. ijrobp.2009.02.006

58. Afkhami Ardekani M, Ghaffari H. Optimization of prostate brachytherapy techniques with polyethylene glycol-based hydrogel spacers: a systematic review. Brachytherapy. 2020;19(1):13-23. doi:10.1016/j.brachy.2019.08.009

59. Giesler RB, Miles BJ, Cowen ME, Kattan MW. Assessing quality of life in men with clinically localized prostate cancer: development of a new instrument for use in multiple settings. Qual Life Res. 2000;9(6):645-665.

60. Sosnowski R. Basic issues concerning health-related quality of life. Cent Eur J Urol. 2017;70(2):206-211. doi:10.5173/ceju.2017.923

61. Gandaglia G, Bray F, Cooperberg MR, et al. Prostate cancer registries: current status and future directions. Eur Urol. 2016;69(6):998-1012. doi:10.1016/j.eururo.2015.05.046

62. Soni PD, Hartman HE, Dess RT, et al. Comparison of populationbased observational studies with randomized trials in oncology. $J$ Clin Oncol. 2019;37(14):1209-1216. doi:10.1200/JCO.18.01074

63. Resnick MJ, Koyama T, Fan K-H, et al. Long-term functional outcomes after treatment for localized prostate cancer. $N$ Engl J Med. 2013;368(5):436-445. doi:10.1056/NEJMoa1209978

64. Barocas DA, Alvarez J, Resnick MJ, et al. Association between radiation therapy, surgery, or observation for localized prostate cancer and patient-reported outcomes after 3 years. JAMA. 2017;317(11):1126-1140. doi:10.1001/jama.2017.1704

65. Chen RC, Basak R, Meyer A-M, et al. Association between choice of radical prostatectomy, external beam radiotherapy, brachytherapy, or active surveillance and patient-reported quality of life among men with localized prostate cancer. JAMA. 2017;317 (11):1141-1150. doi:10.1001/jama.2017.1652

66. Whiting PF, Moore THM, Jameson CM, et al. Symptomatic and quality-of-life outcomes after treatment for clinically localised prostate cancer: a systematic review. BJU Int. 2016;118(2):193204. doi:10.1111/bju.13499

67. Donovan JL, Hamdy FC, Lane JA, et al. Patient-reported outcomes after monitoring, surgery, or radiotherapy for prostate cancer. $N$ Engl J Med. 2016;375(15):1425-1437. doi:10.1056/ NEJMoa1606221
68. Stranne J, Brasso K, Brennhovd B, et al. SPCG-15: a prospective randomized study comparing primary radical prostatectomy and primary radiotherapy plus androgen deprivation therapy for locally advanced prostate cancer. Scand J Urol. 2018;52(56):313-320. doi:10.1080/21681805.2018.1520295

69. Wolff D, Stieler F, Welzel G, et al. Volumetric modulated arc therapy (VMAT) vs. serial tomotherapy, step-and-shoot IMRT and 3D-conformal RT for treatment of prostate cancer. Radiother Oncol. 2009;93(2):226-233. doi:10.1016/j.radonc.2009.08.011

70. Lennernäs B, Majumder K, Damber J-E, et al. Radical prostatectomy versus high-dose irradiation in localized/locally advanced prostate cancer: a Swedish multicenter randomized trial with patient-reported outcomes. Acta Oncol. 2015;54(6):875-881. doi:10.3109/0284186X.2014.974827

71. Crook JM, Gomez-Iturriaga A, Wallace K, et al. Comparison of health-related quality of life 5 years after SPIRIT: surgical prostatectomy versus interstitial radiation intervention trial. J Clin Oncol. 2010. doi:10.1200/JCO.2010.31.7305

72. Giberti C, Chiono L, Gallo F, Schenone M, Gastaldi E. Radical retropubic prostatectomy versus brachytherapy for low-risk prostatic cancer: a prospective study. World J Urol. 2009;27(5):607612. doi:10.1007/s00345-009-0418-9

73. Rodda S, Tyldesley S, Morris WJ, et al. ASCENDE-RT: an analysis of treatment-related morbidity for a randomized trial comparing a low-dose-rate brachytherapy boost with a dose-escalated external beam boost for high- and intermediate-risk prostate cancer. Int J Radiat Oncol. 2017;98(2):286-295. doi:10.1016/j. ijrobp.2017.01.008

74. Hoffman KE, Penson DF, Zhao Z, et al. Patient-reported outcomes through 5 years for active surveillance, surgery, brachytherapy, or external beam radiation with or without androgen deprivation therapy for localized prostate cancer. JAMA. 2020;323(2):149. doi:10.1001/jama.2019.20675

75. Dacal K, Sereika SM, Greenspan SL. Quality of life in prostate cancer patients taking androgen deprivation therapy: quality of life in men with prostate cancer. J Am Geriatr Soc. 2006;54 (1):85-90. doi:10.1111/j.1532-5415.2005.00567.x

76. Cheung AS, de Rooy C, Hoermann R, Lim Joon D, Zajac JD, Grossmann M. Quality of life decrements in men with prostate cancer undergoing androgen deprivation therapy. Clin Endocrinol (Oxf). 2017;86(3):388-394. doi:10.1111/cen.13249

77. Gay HA, Sanda MG, Liu J, et al. External beam radiation therapy or brachytherapy with or without short-course neoadjuvant androgen deprivation therapy: results of a multicenter, prospective study of quality of life. Int J Radiat Oncol. 2017;98(2):304317. doi:10.1016/j.ijrobp.2017.02.019

78. Jenkins DK, Swanson GP, Jhavar SG, Wagner KR, Ha CS, Chen W. Worsening of Urinary Quality of Life (QOL) is seen in patients receiving delayed post-prostatectomy radiation. Int J Radiat Oncol. 2018;102(3):e121. doi:10.1016/j.ijrobp.2018.07.325

79. Melotek JM, Liao C, Liauw SL, Gao X. Quality of life after postprostatectomy intensity modulated radiation therapy: pelvic nodal irradiation is not associated with worse bladder, bowel, or sexual outcomes. Gao X, ed. PLoS One. 2015;10(10):e0141639. doi:10.1371/journal.pone.0141639

80. van Stam M-A, Aaronson NK, Pos FJ, et al. The effect of salvage radiotherapy and its timing on the health-related quality of life of prostate cancer patients. Eur Urol. 2016;70(5):751-757. doi:10.1016/j.eururo.2016.03.010

81. Eastham James A, Kattan Michael W, Eamonn R, et al. Risk factors for urinary incontinence after radical prostatectomy. J Urol. 1996;156(5):1707-1713. doi:10.1016/S0022-5347(01)65488-0

82. Gousse Angelo E, Shahar M, Marie-May L, Fishman Irving J. Artificial urinary sphincter for post-radical prostatectomy urinary incontinence: long-term subjective results. J Urol. 2001;166 (5):1755-1758. doi:10.1016/S0022-5347(05)65668-6 
83. Rehder P, Gozzi C. Transobturator sling suspension for male urinary incontinence including post-radical prostatectomy. Eur Urol. 2007;52(3):860-867. doi:10.1016/j.eururo.2007.01.110

84. Burgio Kathryn L, Stutzman Ray E, Engel Bernard T. Behavioral training for post-prostatectomy urinary incontinence. J Urol. 1989;141(2):303-306. doi:10.1016/S0022-5347(17)40747-6

85. Smit SG, Heyns CF. Management of radiation cystitis. Nat Rev Urol. 2010;7(4):206-214. doi:10.1038/nrurol.2010.23

86. Lee S-I, Park Y-A, Sohn S-K. Formalin application for the treatment of radiation-induced hemorrhagic proctitis. Yonsei Med J. 2007;48(1):97-100. doi:10.3349/ymj.2007.48.1.97

87. Seow-Choen F, Goh H-S, Eu K-W, Ho Y-H, Tay S-K. A simple and effective treatment for hemorrhagic radiation proctitis using formalin. Dis Colon Rectum. 1993;36(2):135-138. doi:10.1007/ BF02051168

88. Tam W, Moore J, Schoeman M. Treatment of radiation proctitis with argon plasma coagulation. Endoscopy. 2000;32(9):667-672. doi:10.1055/s-2000-9020

89. Zhou C, Adler DC, Becker L, et al. Effective treatment of chronic radiation proctitis using radiofrequency ablation. Therap $A d v$ Gastroenterol. 2009;2(3):149-156. doi:10.1177/1756283X081 03341

90. Pinto A, Fidalgo P, Cravo M, et al. Short chain fatty acids are effective in short-term treatment of chronic radiation proctitis. Dis Colon Rectum. 1999;42(6):788-795. doi:10.1007/BF02236937

91. Corman JM, McCLURE D, Pritchett R, Kozlowski P, Hampson NB. Treatment of radiation induced hemorrhagic cystitis with hyperbaric oxygen. J Urol. 2003;169(6):2200-2202. doi:10.109 7/01.ju.0000063640.41307.c9

92. Woo TCS, Joseph D, Oxer H. Hyperbaric oxygen treatment for radiation proctitis. Int $J$ Radiat Oncol. 1997;38(3):619-622. doi:10.1016/S0360-3016(97)00017-5

93. Incrocci L, Slob AK, Levendag PC. Sexual (dys)function after radiotherapy for prostate cancer: a review. Int $J$ Radiat Oncol. 2002;52(3):681-693. doi:10.1016/S0360-3016(01)02727-4

94. Chung E, Brock G. Sexual rehabilitation and cancer survivorship: a state of art review of current literature and management strategies in male sexual dysfunction among prostate cancer survivors. J Sex Med. 2013;10(S1):102-111. doi:10.1111/j.1743-6109.2012. 03005.x

95. Zelefsky Michael J, Daniel S, Dunn BR, et al. Prophylactic sildenafil citrate improves select aspects of sexual function in men treated with radiotherapy for prostate cancer. $J$ Urol. 2014;192(3):868-874. doi:10.1016/j.juro.2014.02.097
96. Mazzola C, Mulhall JP. Penile rehabilitation after prostate cancer treatment: outcomes and practical algorithm. Urol Clin North Am. 2011;38(2):105-118. doi:10.1016/j.ucl.2011.03.002

97. Dubocq FM, Bianco FJ, Maralani SJ, Forman JD, Dhabuwala CB. Outcome analysis of penile implant surgery after external beam radiation for prostate cancer. J Urol. 1997;158(5):17871790. doi:10.1016/S0022-5347(01)64129-6

98. Pahlajani G, Raina R, Jones S, Ali M, Zippe C. Vacuum erection devices revisited: its emerging role in the treatment of erectile dysfunction and early penile rehabilitation following prostate cancer therapy. J Sex Med. 2012;9(4):1182-1189. doi:10.1111/ j.1743-6109.2010.01881.x

99. Cormie P, Newton RU, Taaffe DR, Spry N, Galvão DA. Exercise therapy for sexual dysfunction after prostate cancer. Nat Rev Urol London. 2013;10(12):731-736. doi:10.1038/nrurol.2013.206

100. Badr H, Taylor CLC. Sexual dysfunction and spousal communication in couples coping with prostate cancer. Psychooncology. 2009;18(7):735-746. doi:10.1002/pon.1449

101. Delobel J-B, Gnep K, Ospina JD, et al. Nomogram to predict rectal toxicity following prostate cancer radiotherapy. Sung S-Y, ed. PLoS One. 2017;12(6):e0179845. doi:10.1371/journal.pone. 0179845

102. Valdagni R, Rancati T, Fiorino C, et al. Development of a set of nomograms to predict acute lower gastrointestinal toxicity for prostate cancer 3D-CRT. Int J Radiat Oncol. 2008;71(4):10651073. doi:10.1016/j.ijrobp.2007.11.037

103. Valdagni R, Kattan MW, Rancati T, et al. Is it time to tailor the prediction of radio-induced toxicity in prostate cancer patients? Building the first set of nomograms for late rectal syndrome. Int $J$ Radiat Oncol. 2012;82(5):1957-1966. doi:10.1016/j.ijrobp.2011. 03.028

104. Mathieu R, Arango JDO, Beckendorf V, et al. Nomograms to predict late urinary toxicity after prostate cancer radiotherapy. World J Urol. 2014;32(3):743-751. doi:10.1007/s00345-013-11 46-8

105. Montie JE. Counseling the patient with localized prostate cancer. Urology. 1994;43:36-40. doi:10.1016/0090-4295(94)90216-X

106. Valicenti RK, Gomella LG, El-Gabry EA, et al. The multidisciplinary clinic approach to prostate cancer counseling and treatment. Semin Urol Oncol. 2000;18(3):188-191.

107. Gomella LG, Lin J, Hoffman-Censits J, et al. Enhancing prostate cancer care through the multidisciplinary clinic approach: a 15year experience. J Oncol Pract. 2010;6(6):e5-e10. doi:10.1200/ JOP.2010.000071

\section{Publish your work in this journal}

Research and Reports in Urology is an international, peer-reviewed, open access journal publishing original research, reports, editorials, reviews and commentaries on all aspects of adult and pediatric urology in the clinic and laboratory including the following topics: Pathology, pathophysiology of urological disease; Investigation and treatment of urological disease; Pharmacology of drugs used for the treatment of urological disease. The manuscript management system is completely online and includes a very quick and fair peer-review system, which is all easy to use. Visit http://www.dovepress.com/ testimonials.php to read real quotes from published authors 